\title{
Fundamentos Pedagógicos para la enseñanza- aprendizaje de la Educación Física en Modalidad Virtual: Un reto actual
}

Pedagogical foundations for the teaching learning of physical education in a virtual modality: a current challenge

Orlando Rodrigo Carrasco Coca. ${ }^{1}$, José Guillermo Caicedo Merizalde. ${ }^{2}$, Ower Armando Savedra Valdiviezo. ${ }^{3} \&$ Vanessa Lucia Ochoa Sangurima. ${ }^{4}$

Abstract.

DOI: $\underline{\text { https://doi.org/10.33262/cienciadigital.v5i1.1542 }}$

Introduction. The teaching-learning process continues at the different levels and subjects in times of COVID 19, it has demanded to go from the face-to-face mode to the virtual one. In Physical Education, this has had a considerable impact. Objective. To reflect on the importance of the applicability of the pedagogical foundations for the teaching - learning of Physical Education in the virtual modality. Methodology. The study followed a descriptive, non-experimental methodology, using theoretical methods based on the RSL (Systematic Review of Literature), making it possible to consult 25 sources related to the research topic. Results.1.- It was possible to identify 15 potentially relevant studies that contributed to systematizing the strategies followed on the importance of the applicability of the pedagogical foundations of Physical Education for its teaching-learning through the virtual modality. 2. The analysis reveals the challenge that this has meant, precisely because Physical Education is contemplated within the group of practical subjects and the limited competencies of teachers to

\footnotetext{
${ }^{1}$ Universidad de las Fuerzas Armadas. (ESPE), Carrera: Pedagogía de la Actividad Física y Deporte orcarrasco@espe.edu.ec ORCID ID: https://orcid.org/0000-0001-6995-2534

${ }^{2}$ Universidad de las Fuerzas Armadas. (ESPE), Carrera: Pedagogía de la Actividad Física y Deporte jgcaicedo@espe.edu.ec ORCID ID: https://orcid.org/0000-0001-5772-3304

${ }_{3}^{3}$ Universidad de las Fuerzas Armadas. (ESPE), Carrera: Pedagogía de la Actividad Física y Deporte oasaavedra1 @espe.edu.ec ORCID ID: https://orcid.org/0000-0003-3000-4662

${ }^{4}$ Universidad de las Fuerzas Armadas. (ESPE), Carrera: Pedagogía de la Actividad Física y Deporte vane4lali@gmail.com_ORCID ID: https://orcid.org/0000-0003-3928-118
} 
develop the teaching-learning process in this new scenario. 3. The opportunities offered by the TICs have allowed continuity in the teaching-learning process and have a positive impact on the development of other competencies in both teachers and students. Conclusions. The analysis of the preceding theories confirm the importance of the pedagogical foundations that support Physical Education and its usefulness for the development of the teaching-learning process through the virtual modality, while being aware that the main thing is not technology as such, but the use we can make of it within the educational process. Regardless of this, migrating from the face-to-face mode to the virtual one has been a great challenge, causing the need to promote in record time the development of teaching-methodological skills and abilities in teachers and students.

Keywords: virtual modality, physical education, teaching-learning process, pedagogical foundations.

\section{Resumen.}

Introducción. Continuar el proceso de enseñanza aprendizaje en los diferentes niveles y asignaturas en tiempos de COVID 19, ha demandado pasar de la modalidad presencial a la virtual. En la Educación Física esto ha tenido una repercusión considerable. Objetivo Reflexionar sobre la importancia de la aplicabilidad de los fundamentos pedagógicos para la enseñanza - aprendizaje de la Educación Física en la modalidad virtual. Metodología. El estudio siguió una metodología descriptiva, no experimental, mediante el empleo de métodos teóricos con base en la RSL (Revisión Sistemática de Literatura), posibilitando consultar 25 fuentes relacionadas con el tema de investigación. Resultados.1.- Se logró la identificación de 15 estudios potencialmente relevantes que contribuyeron a sistematizar las estrategias seguidas sobre la importancia de la aplicabilidad de los fundamentos pedagógicos de la Educación Física para su enseñanza-aprendizaje mediante la modalidad virtual. 2. El análisis devela el reto que esto ha significado, precisamente por estar contemplada la Educación Física dentro del grupo de asignaturas prácticas y las limitadas competencias de los docentes para desarrollar el proceso de enseñanza -aprendizaje en este nuevo escenario. 3. Las oportunidades que ofrecen las TICs, ha permitido dar continuidad al proceso de enseñanza - aprendizaje e incidir de manera positiva en el desarrollo de otras competencias tanto en los docentes como en los estudiantes. Conclusiones: El análisis de las teorías precedentes confirman la importancia de los fundamentos pedagógicos que sustentan la Educación Física y su utilidad para el desarrollo del proceso de enseñanza-aprendizaje mediante la modalidad virtual, sin dejar de ser conscientes que lo primordial no es la tecnología como tal, sino el uso que podemos hacer de ella dentro del proceso educativo. Independientemente a ello migrar 
de la modalidad presencial a la virtual, ha constituido un gran reto, provocando la necesidad de potenciar en tiempo récord el desarrollo de competencias y habilidades didácticas -metodológicas en los docentes y estudiantes.

Palabras claves: Educación Física, proceso de enseñanza-aprendizaje, fundamentos pedagógicos, modalidad virtual.

\section{Introducción.}

La pandemia generada a nivel mundial y en la República de Ecuador en particular, por la incidencia del Covid-19, ha obligado al confinamiento social para resguardar la salud e integridad de todos los ciudadanos. Lo anterior ha tenido una extraordinaria repercusión en el contexto educativo. Tanto los docentes como los estudiantes nos hemos visto en la necesidad de aprender nuevas formas de cómo enseñar y cómo aprender. Ello además de ser un gran reto, ha traído modificaciones en los estilos de enseñanza-aprendizaje en las diferentes disciplinas, incluyendo la Educación Física.

En este orden se debe considerar que la migración de la modalidad presencial a la virtual dada las propias características de la Educación Física, ha obligado a los docentes a desarrollar competencias profesionales en tiempo récord, que les permitan entre otros aspectos utilizar los recursos tecnológicos que conforman las Tecnologías de la Información y Comunicación (TICs), para así dar continuidad al proceso de enseñanza-aprendizaje en todos los niveles con la calidad requerida. Ello ha generado múltiples preocupaciones, unidas al surgimiento de innumerables acciones a desarrollar durante este período; por supuesto sin dejar de tener en cuenta los fundamentos pedagógicos y los presupuestos teóricos-prácticos para la enseñanza de la Educación Física.

Ante todo, esto los docentes de Educación Física se han visto en la necesidad de evaluar varias estrategias y procedimientos didácticos posibles a aplicar en esta modalidad virtual, que además de permitir la continuidad del proceso de enseñanza - aprendizaje, manteniendo los fundamentos pedagógicos establecidos, faciliten lograr de manera progresiva la concreción de resultados de aprendizajes significativos por parte de los estudiantes. Respecto a ello, es muy interesante considerar los aportes de Rodríguez, Peña, Reginfo y Rojas, (2015), los cuales han señalado que el tratamiento de la Educación Física, ya sea a partir la visión del docente como del estudiante, debe abordarse desde diferentes perspectivas; enfatizando en la importancia sobre la comprensión y visión en relación con los procesos metodológicos, los cuales según los autores precedentes deben estar orientados a la investigación y aplicación del dominio de las TICs en la Educación Física. Indican además que se debe combinar esquemas que posean un carácter cualitativo y cuantitativo, de manera tal que se sistematice 
la experiencia de los docentes en función de la complejidad del proceso de enseñanzaaprendizaje.

En esta misma línea de pensamiento varios autores han realizado estudios relacionados con la búsqueda de estrategias metodológicas óptimas que permitan dar respuesta a la nueva situación a que nos enfrentamos, para lo cual se sistematizan las teorías en relación con la aplicabilidad de las clases virtuales como la vía principal para lograrlo, incluyendo en esto las clases de Educación Física. Siguiendo estas ideas Alfonso, Enríquez, Alcívar, (2020), explican que resulta necesario, que los docentes cuenten con el dominio de las Tecnologías de Información y Comunicación (TICs) y paralelamente a ello, deben ser capaces de aplicar estrategias didácticas coherentes, para lograr la necesaria efectividad del proceso de enseñanza-aprendizaje, dirigidas, ante todo, a mantener la motivación y el interés en los estudiantes por la clase de Educación Física. Conforme a lo señalado Barahona, (2012), planteó que se necesita una Educación Física que sea capaz de responder a nuevos desafíos, significando en su estudio la necesidad del establecimiento de pautas que nos ayuden a integrarlas como un valor añadido y que enriquezcan los procesos de enseñanza- aprendizaje de la disciplina.

Este nuevo escenario ha influenciado en la realización de estudios relacionados con la continuidad del proceso de enseñanza-aprendizaje, entre ellos podemos citar a Alfonso Enríquez, Alcívar, (2020), quienes en su estudio, evaluaron la adaptación de los docentes a esta nueva modalidad, para lo cual proponen la realización de actividades prácticas que puedan ser realizadas en espacios reducidos y sin los medios y recursos deportivos idóneos, sino con otros similares alternativos existentes en nuestras casas e incorporando a integrantes de la propia familia, propiciando la integración y al mismo tiempo la realización de los ejercicios prácticos de la actividad física.

En esa misma dirección Rodríguez, Trybalski, (2020), realizaron propuestas que aportan al Programa de la Continuidad Pedagógica, enmarcadas estas en los diseños curriculares existentes de la Educación Física, los cuales permiten la posibilidad de su realización por los estudiantes en el hogar, además recogen las actividades realizadas en el periodo, las cuales se registran a través de fotos o videos y enviadas al docente mediante WhatsApp, redes sociales, mail, y otras.

Cabe señalar que un aspecto muy importante es el referido al entorno virtual en la disciplina de Educación Física, dado que debe satisfacer los requerimientos propios de esta disciplina, de manera que sea consecuente con sus fundamentos pedagógicos. Al respecto se concuerda con Úbeda y Molina, (2016), al indicar que un entorno virtual en el área de Educación Física ofrece diversos aspectos positivos, entre los que destacan la posibilidad de conexión de la teoría con la práctica, enfatizando que esto posibilita que los estudiantes puedan debatir de 
manera crítica y reflexiva temas relacionados con la disciplina, además de permitir que puedan utilizar herramientas y recursos tecnológicos de multimedia, promoviendo así la activa participación de los estudiantes en el proceso de enseñanza-aprendizaje.

Todo esto impone tener una mirada diferente al rol del docente, el cual debe prepararse para el logro de un proceso de enseñanza aprendizaje significativo, efectivo y de calidad. Es por ello que se requiere contar con una planta docente capacitada y con las competencias necesarias que le permitan elevar su nivel de desempeño y a su vez lograr los objetivos instructivos y educativos necesarios a alcanzar en cada nivel de enseñanza. Ello introduce además considerar, la incidencia que tiene la Educación Física en el logro de las capacidades básicas, así como el dominio de la competencia digital en función de esta disciplina. En relación a esto, estamos de acuerdo con Reyes \& Fuentes, (2011), al aseverar que un buen uso de las Tecnologías de la Información y Comunicación está estrechamente relacionado a la competencia de aprender a aprender, dado que esta presupone contar con habilidades sobre cómo planificar, desarrollar y evaluar para iniciarse en el aprendizaje y a la vez, continuar aprendiendo de manera eficaz y autónoma en función a los propios objetivos y necesidades del proceso de enseñanza-aprendizaje.

Otro aspecto que debe valorarse de manera profunda al enseñar la Educación Física mediante la modalidad virtual, es el relacionado con el papel integrador en la acción de enseñar valores y actitudes; ya que si bien es cierto era prácticamente fácil de alcanzar en la modalidad presencial, dado que es precisamente a través del juego que los estudiantes aprenden las habilidades sociales, en condiciones de no presencialidad se requiere pensar y desarrollar acciones sobre cómo lograrlo. Por lo que el docente debe trazar las estrategias didácticas necesarias para que, dentro del proceso de enseñanza de la Educación Física en la modalidad virtual, pueda mantenerse también el carácter lúdico, e ir incorporando las acciones necesarias que permitan lograr el desarrollo de valores. Para ello resulta fundamental el perfeccionamiento de la creatividad como competencia esencial a alcanzar por los docentes.

Sin embargo, independientemente de los avances logrados y de los retos que ha impuesto la no presencialidad en las clases de Educación Física, es fundamental comprender la estrecha relación que debe estar presente entre los fundamentos pedagógicos para la enseñanzaaprendizaje de la Educación Física y el nivel de competencias que debe poseer la planta docente sobre cómo aplicarlos en la modalidad virtual. Derivado de lo anterior resulta transcendental reflexionar sobre la importancia de la aplicabilidad de los fundamentos pedagógicos para la enseñanza - aprendizaje de la Educación Física en la modalidad virtual, para la consecución de los fines instructivos y educativos de la Educación Física, constituyéndose así el objetivo general del presente estudio. 


\subsection{Fundamentos Pedagógicos Generales para la Enseñanza-Aprendizaje de la Educación Física.}

Los cambios que se han venido dando en el decursar histórico social han tenido gran influencia en los procesos de enseñanzas -aprendizajes. Actualmente la Pedagogía de la Educación Física Contemporánea, promueve la participación en la acción formativa de todas las energías del estudiante, las relativas a las físicos-sensoriales, así como, las psíquicoespirituales, pretendiendo al mismo tiempo desarrollar un efecto formativo y cultural más allá de la transmisión de habilidades y de sus acciones en favor de la Comunidad. En ese contexto, la Pedagogía de la Educación Física, se encamina a lograr la participación del estudiante, en forma integral en la acción formativa, ello implica plantearse ser una Pedagogía orientada al estudiante, en la que es considerado un sujeto activo de su aprendizaje. Esta nueva visión pedagógica se centra en una Pedagogía integradora y diferenciada que incluye el conocimiento del estudiante, sobre el desarrollo cognitivo, afectivo y motriz, reconociendo que cada estudiante es un ser diferente, con necesidades e intereses distintos y con su propio ritmo de aprendizaje.

Al respecto Maqueira, et al., (2018), enfatizan en la importancia de la Pedagogía No directiva para la consecución de los objetivos instructivos y educativos de la clase de Educación Física, señalando que la Educación Física Contemporánea debe estar sustentada sobre la base de la introducción de nuevas formas de enseñanza-aprendizaje que permitan dar respuesta a la diversidad de estudiantes y al mismo tiempo facilitar la concreción de aprendizajes significativos.

La literatura precedente destaca además la importancia de los fundamentos pedagógicos generales para la enseñanza - aprendizaje de la Educación Física. Relacionando dentro de estos los siguientes:

- Fundamento de la adaptación de la naturaleza humana: Esta referido a la realización adecuada de los ejercicios necesarios para la formación y el fortalecimiento de los órganos. Mucho ejercicio lo perjudica y poco lo atrofia. También el que se relaciona con la alternancia entre estímulos e intervalo de descanso durante la realización del ejercicio físico, así como tener presente que la persona cuando actúa lo hace integralmente (aspecto motor, cognitivo y socio-afectivo).

- Fundamento de adaptación a la evolución: El niño al convertirse en adolescente pasa por diferentes etapas en el desarrollo de sus capacidades y habilidades, tanto en el aspecto cognitivo, motor y afectivo. Las actividades de Educación Física deben adecuarse a estos cambios.

- Fundamento de la individualidad: El ser humano es único, ninguno es igual a otro, por lo que debe tenerse en cuenta el ritmo personal, el desempeño individual, su 
capacidad de rendimiento, así como las necesidades, intereses y demandas culturales de cada estudiante en particular.

- Fundamento de la solidaridad: El ser humano es un ente social, o sea siempre vive y se desarrolla en comunidad, ello exige practicar la tolerancia, el respeto mutuo y las reglas de convivencia: cooperación, amabilidad y otros.

- Fundamento de la totalidad: El ser humano es un todo. Este fundamento se observa desde el punto de vista antropológico, didáctico y metodológico en la concepción del movimiento.

- Fundamento de la realidad: No debemos guiarnos solo por la teoría, debemos también observar las actividades que realizan los estudiantes, sus intereses lúdicos, recreativos y deportivos.

- Fundamento de la creatividad: Las actividades lúdicas, deportivas, recreativas, artísticas, culturales y manuales, generan espacios para cultivar la creatividad.

- Fundamento de la espontaneidad: Las tareas asignadas a los estudiantes, deben estar diseñadas de manera que los estudiantes puedan realizarlas con sus propias fuerzas, dejándoles la libertad para seguir su camino elegido, así como para determinar libremente su meta.

En este contexto un aspecto primordial lo constituyen las Competencias Básicas (CCBB), como instrumento de formación y preparación de los estudiantes para su futuro. Coincidimos con Monzonis, (2015) al expresar que las mismas buscan conseguir una visión alternativa de la educación, abarcando el quehacer diario de las clases para formar estudiantes competentes en su futuro.

De igual modo se coincide con Ruiz, (2010), al aseverar que dentro del marco de las Competencias Básicas la Educación Física un rol fundamental e integrador se ubica en el momento de enseñar valores y actitudes, o sea, cuando en clase se imparten los contenidos del currículo para alcanzar los objetivos de la disciplina, a través del juego los estudiantes aprenderán habilidades sociales (aceptar las normas de juego, cooperar, ser solidarios, dialogar, escuchar, ser responsables, comprometerse y otras) y códigos de conductas que posteriormente transferirán a su vida cotidiana. Por otra parte, y en concordancia con García, (2010), a través de la Educación Física, los estudiantes, podrán adquirir hábitos saludables, así como que les suministrará de los medios y conocimientos necesarios para el disfrute del ocio y del tiempo libre, conduciéndolos a una mejora en su calidad de vida.

Todo ello nos confirma el elevado grado de aportación en la consecución de las Capacidades Básicas que tiene la disciplina de Educación Física en las competencias de carácter práctico, en esta dirección estamos de acuerdo con los estudios realizados por Barrachina \& Blazco, (2012), al demostrar que el docente de Educación Física incide mayormente en los estudiantes, en la aportación en las competencias de carácter práctico, o sea, las competencias 
de aprender a aprender, autonomía e iniciativa personal, interacción con el mundo físico y social y la social y ciudadana, en comparación con aquellas que tienen un carácter más teórico, tales como la lingüística, la matemática, la cultural y artística y la de tratamiento de la información y de competencia digital.

En correspondencia con lo señalado y sistematizando la importancia de los fundamentos pedagógicos que sustentan el proceso de enseñanza-aprendizaje en la clase de Educación Física tanto para la modalidad presencial como la virtual, somos del criterio que los aportes de Úbeda y Molina, (2016), seguidos por Rodríguez, Trybalski, (2020) y Alfonso Enríquez, Alcívar, (2020), han permitido develar el gran reto que ha impuesto a la comunidad educativa relacionada con la Educación Física la presencia del Covid 19 y su impacto en el desarrollo de estrategias educativas conducentes al logro de aprendizajes significativos en los estudiantes. Esto ha implicado reflexionar respecto a la necesidad de que imperiosamente deben ser antepuestos los procesos pedagógicos sobre los tecnológicos y eliminar la creencia de que existe una incompatibilidad entre la tecnología y la práctica física, por lo cual debemos ser consciente que lo primordial no es la tecnología como tal, sino el uso que podemos hacer de ella dentro del proceso educativo, para lo cual el docente debe poseer las competencias requeridas.

En atención a lo señalado cabe comentar que, si bien es cierto que con la llegada del Covid 19, se ha requerido modificar las estrategias de enseñanza-aprendizaje también se ha podido demostrar que los fundamentos pedagógicos continúan siendo esenciales dentro del proceso de clase de Educación. Estos constituyen la base para alcanzar los objetivos instructivos y educativos en cada clase y nivel de enseñanza.

\subsection{La Enseñanza -Aprendizaje de la Educación Física en la Modalidad Virtual. Retos actuales.}

La utilización de la modalidad virtual, dentro del proceso de enseñanza-aprendizaje se ha hecho un imperativo de nuestros días debido a la pandemia del Covid-19, y ello se realiza de forma generalizada a todas las asignaturas de las diferentes carreras, ya tengan un componente práctico mayor o menor. En este contexto se incorpora la disciplina de Educación Física, que por su propia concepción necesita de una estrecha vinculación de actividades teóricas y prácticas que conlleven al óptimo cumplimiento del proceso educativo y de la obtención de los resultados esperados conforme a los objetivos de desempeño a lograr por los estudiantes de la disciplina.

En relación a ello, es importante remarcar que, a diferencia de otras disciplinas, la enseñanza de la Educación Física a través de la modalidad virtual tiene muchos detractores, existiendo una actitud reacia a su utilización por parte de muchos docentes, dado a supuesta aseveración de que con ello se pierde la esencia del aprendizaje físico y que no se cumplirían con los 
objetivos del proceso de enseñanza- aprendizaje. Respecto a ello varios autores han señalado estos criterios, como es el caso de Ferreres, (2011), quien expresó que el poco uso de las Tecnologías de Comunicación e Información (TICs), está dada en la creencia por algunos docentes, en que la implementación tecnológica va a significar una disminución del compromiso motor de los estudiantes, pues supone una reducción del volumen de actividad física, su naturaleza ludo-práctica o su tradición experiencial.

En estudios realizados por diversos autores sobre el comportamiento del proceso de enseñanza-aprendizaje y la utilización de las TICs, en la Educación Física se ha constatado la poca utilización de los recursos y herramientas digitales que esta modalidad ofrece. Al respecto podemos citar a Moreira, (2018), quien plantea que los docentes de Educación Física utilizan con mayor incidencia las aplicaciones de mensajería instantánea para impartir sus clases, dado que permiten una mejor comunicación entre docentes- estudiantes, por su parte (Salinas, 2017), plantea el intercambio de mensajes de textos, documentos, videos, imágenes y audios, que contribuye al desarrollo del proceso de enseñanza-aprendizaje, pero no obstante, deja grandes vacíos que pudieran completarse con el desarrollo de sesiones, aplicaciones de organización y evaluación. En este mismo ámbito Moreno \& Trigo, (2017), explican, que ello corrobora que los docentes se limitan en el uso de los recursos digitales, teniendo la posibilidad de utilizar una gran variedad de medios y recursos tecnológicos que pueden ser utilizados por el docente en el desarrollo del proceso de enseñanza-aprendizaje.

No obstante a lo señalado y debido al propio desarrollo que ha experimentado en los últimos años la aplicabilidad de las TICs en todos las áreas del proceso educativo, podemos afirmar que también se ha incursionado con ellas en la enseñanza de la Educación Física, en relación a ello Cazorla, Ibánez, (2018), señalan que ha de considerarse que las TICs, ya se están utilizando para realizar actividad física, como es la utilización de smartphone para obtener datos informativos sobre la práctica de esta actividades, para conocer los esfuerzos realizados en las caminatas diarias y su equivalente en gastos calóricos entre otros y en cuanto a su uso en la Educación Física, podemos encontrar diversos estudios que afirman sobre el aporte de diferentes aspectos positivos que permite ampliar las posibilidades metodológicas en la enseñanza de la disciplina y la buena acogida por parte de los estudiantes.

Es por ello que, en la obtención del óptimo resultado del proceso de enseñanza- aprendizaje en esta disciplina, mediante la modalidad virtual que implica la inclusión de las Tecnologías de la Información y Comunicación, exigen del docente un elevado nivel de desempeño y competencias, unida a una adecuada preparación, dado que en ella deben confluir los aspectos relacionados propiamente con los fundamentos pedagógicos y teóricos-prácticos de la disciplina de Educación Física, así como la utilización efectiva de las herramientas tecnológicas y los espacios virtuales asociados a esta modalidad. Sin dejar atrás el aspecto 
del desarrollo pedagógico didáctico de los docentes, que lleva implícito el logro de un aprendizaje significativo en los estudiantes.

Al respecto coincidimos con Romero \& Kabusch, (2017), cuando plantean que la inclusión de las Tecnologías de la Información (TICs) para abordar situaciones en el sistema educativo, no se refiere solo a la incorporación como tal de las mismas, sino que debe constituir un reflejo de la institucionalidad de logros en la construcción de los conocimientos.

En concordancia con lo explicado, un aspecto fundamental es el referido a los espacios virtuales, que en nuestro caso lo constituye el aula virtual, que conforme a Florez \& Ramos, (2015) indica que no solo debe constituir un mecanismo para la distribución de la información, sino que además el sistema tiene que ser capaz de dar cabida a las actividades involucradas en el proceso de enseñanza-aprendizaje, o sea las relacionadas a la interactividad, comunicación, aplicación de los conocimientos, evaluación y el manejo de la clase.

En esta dirección Romero \& Kabusch, (2017), expresan que la estructura de un entorno o aula virtual debe contar con espacios para divulgar y debatir materiales teóricos y/o prácticos, por parte de los profesores y estudiantes; espacios para la actividad del estudiante, donde le permita enviar y almacenar informes y clases realizadas; espacios de intervención de docente, donde pueda aportar materiales, para dar seguimiento a los estudiantes y otros; espacios para la colaboración y consulta tales como foros y chats.

Lo explicado hace coincidir con Pannesi, (2011) al enunciar que la Educación Física, debe aprovechar las Tecnologías de la Información y Comunicación para lograr ambientes enriquecidos de aprendizaje, de manera que se haga más atractiva su enseñanza y a la vez contribuya a que se produzca un cambio metodológico, que varíe las prácticas pedagógicas tradicionales fundamentadas en la rigidez de los contenidos, el monologo docente y el exacerbado protagonismo del libro de texto hacia prácticas más activas, humanas y desarrolladoras.

No obstante, a ello, también debemos tener claro que las experiencias virtuales siempre estarán basadas en el enriquecimiento de las vivencias reales, porque conforme a Barahona, (2012), no se puede olvidar que la Educación Física es en esencia la Pedagogía motriz del aprender haciendo y aprender reflexionando sobre la acción. Por lo que el uso de la modalidad virtual, o sea de las Tecnologías de la Información y la Comunicación, debe estimular los principios del desarrollo al máximo en los estudiantes del potencial físico, intelectual y socio-afectivo. 
De igual forma, el uso de la modalidad virtual de esta disciplina, debe por sobre todo, respetar los fundamentos pedagógicos generales para la enseñanza - aprendizaje de la Educación Física, de manera que no se pierda, durante la impartición de la misma por esta modalidad la propia esencia de la disciplina, que como se ha expuesto anteriormente, dado su propia concepción necesita de una estrecha vinculación de actividades teóricas y prácticas que conlleven al cumplimiento y óptimo desempeño del proceso de enseñanza-aprendizaje. En este mismo orden es importante remarcar también que la modalidad virtual cuenta consigo con una significativa ventaja comparada con la modalidad presencial y es la referente a la disponibilidad del espacio-temporal, que constituye de por si una condicionalidad fundamental en el proceso de enseñanza aprendizaje de la disciplina, dado que no existe limitaciones de espacio ni de tiempo.

Respecto a esta cuestión compartimos con Díaz (2011), al referirse al tema, citando como ejemplo que la modalidad virtual facilita estar en contacto con entornos virtuales de comunicación y aprendizajes, donde el espacio no se limita a un lugar concreto: escuela, patio, o gimnasio, al contrario, permite que se desarrolle e involucren otros espacios, sin límites de tiempo, disponiendo así de espacios de aprendizajes fuera del contexto áulico o escolar.

Por su parte, Rojas, (2018) y Fariña, González, \& Área (2013), en sus estudios, aportan sobre la creación de un aula virtual de aprendizaje para el área de Educación Física, la cual permitió la interacción entre el docente y los estudiantes, con el propósito de contribuir al desarrollo del componente cognitivo de las competencias específicas de esta disciplina, recurriendo al uso del soporte de diferentes herramientas TICs, que usadas complementariamente en la plataforma LMS choology, permitían además un uso más eficiente de los dispositivos (smartphone, computadores y tablets, de internet, a los que los estudiantes tienen acceso.

En un estudio similar realizado por Sánchez, Ávila, García y Bravo, (2020), a partir de los resultados obtenidos, pudieron concluir que los docentes de Educación Física, utilizan varios estilos de enseñanza en sus clases virtuales, no obstante, también se restringen al aplicar los recursos digitales disponibles, reduciendo las posibilidades de aprendizaje de los estudiantes.

Se comparte con los autores precedentes que, en vista a mantener los fundamentos pedagógicos de la Educación Física en su modalidad virtual, se hace necesario que los docentes desarrollen su carácter creativo, de manera que principalmente mantenga el componente lúdico de esta disciplina, en el cual se basa en un alto grado el desarrollo del proceso de enseñanza-aprendizaje. Un ejemplo de ello pudiera ser la creación de juegos interactivos en plataformas informáticas, para recuperar las habilidades motrices del estudiante, las cuales se encuentran dentro de los contenidos propios de las clases 
presenciales, dándosele al mismo tiempo continuidad a lo que se recoge en los diseños curriculares.

Paralelamente a ello, el docente que imparte la disciplina de Educación Física, independientemente del conocimiento que tenga de la misma, debe dominar todas las herramientas que permita el óptimo conocimiento de los estudiantes en un aprendizaje virtual, al respecto coincidimos con Becerril, Sosa, Delgadillo y Torres (2015), al plantear que el docente debe ser capaz de implementar estrategias de enseñanza y aprendizaje que posibiliten el trabajo colaborativo y la construcción en una comunidad virtual de aprendizaje.

Todo lo expuesto anteriormente, denota los diferentes retos que deben ser salvados, tanto a nivel nacional como internacional por los docentes de Educación Física en los contextos actuales; donde la presencia del Covid 19 ha provocado migrar de la modalidad presencial a la virtual, derivándose la necesidad de articular la praxis diaria con el cumplimiento de los fundamentos pedagógicos que sustentan la enseñanza-aprendizaje de la Educación Física mediante la modalidad virtual. Es importante significar, además, que para lograr el desarrollo de habilidades y aprendizajes significativos los docentes y los estudiantes deben establecer prioritariamente el desarrollo de sus competencias que les permitan ascender a un eslabón superior en la construcción de los conocimientos y al mismo tiempo en el desarrollo pedagógico didáctico de los docentes de esta disciplina.

\section{Metodologia.}

Para el desarrollo del trabajo se utilizó una metodología descriptiva, no experimental con el empleo de métodos teóricos, dentro de los cuales se encuentran: el histórico-lógico, el analítico - sintético, inductivo -deductivo y la revisión documental; permitiendo así realizar la RSL (Revisión Sistemática de Literatura), según las etapas establecidas las cuales incluyen la planificación, análisis y resultados. La consulta de la literatura precedente contempló un rango de tiempo entre el 2010 y el 2020, consultándose para ello trabajos publicados en bases: tales como Scopus, PubMed, Google Académico, Scielo, Redalcy, Dialnet, Latindex, Web of Science, Publice, incluyendo la valoración de los aportes realizados en Tesis de Doctorado, Maestría y Licenciatura en relación con el tema objeto de estudio.

Para la selección de información y fuentes relevantes se determinaron los criterios de inclusión y exclusión, los cuales permitieron el control de la calidad y el rigor del trabajo presentado.

Criterios de inclusión

1. Tipo de estudios: Estudios de revisiones teóricas o experimentales que sistematicen o desarrollen teorías en relación con los fundamentos pedagógicos y metodológicos 
para la enseñanza-aprendizaje de la Educación Física en condiciones de presencialidad y en modalidad virtual.

2. Tipo de participantes: Docentes, investigadores y estudiantes involucrados con la enseñanza-aprendizaje de la Educación Física.

3. Tipo de resultados: Metodologías, modelos, programas que contemplen resultados en relación con el tema objeto de estudio

4. Tesis de Doctorado o Maestría realizadas en los últimos 10 años en Universidades Internacionales o Nacionales sobre el tema de investigación.

Criterios de exclusión

1. Estudios o investigaciones realizadas en un rango de tiempo mayor al contemplado en la investigación.

2. Artículo en idioma diferente del español o inglés.

3. Otros estudios que por su tema o resultados no aporten elementos reveladores para nuestra investigación.

\section{Resultados.}

El confinamiento que ha generado la actual pandemia de Covid-19, ha traído como consecuencia un desplazamiento de las actividades educativas presenciales a virtuales, lo que ha conllevado de igual manera, a una adaptación en las estrategias educativas de los docentes para enfrentar este nuevo escenario que se suscita a distancia, con la exigencia al mismo tiempo, de lograr en los estudiantes, desempeños adecuados en la formación de las competencias básicas específicas de la Educación Física.

En relación a esto se logra sistematizar que a pesar de que la modalidad virtual, era utilizada en mayor medida en la formación de conocimientos de otras disciplinas académicas y no se comportaba de igual manera en la disciplina de Educación Física, dado el carácter eminentemente práctico de esta disciplina y los criterios antagónicos generalizados sobre la imposibilidad de la aplicación de las TICs en el proceso educativo de la misma, al considerar que supuestamente se pierde ese componente práctico, se ha logrado sistematizar las teorías en relación con los aportes de diferentes autores tales como: Pannesi, (2011), Florez, Ramos, (2015), Úbeda y Molina, (2016), seguidos por Rojas, (2018), Rodríguez, Trybalski, (2020) y Alfonso, et al., (2020), Sánchez, Ávila, García y Bravo, (2020), entre otros, que sí confirman la utilidad de la modalidad virtual para la consecución de los objetivos instructivos y educativos de la Educación Física en relación directa con los fundamentos pedagógicos que la componen. 
$\mathrm{Al}$ respecto se coincide con Ferreras, (2011), al indicar que el poco uso de las Tecnologías de Comunicación e Información (TIC) en la Educación Física, está dada en la creencia por algunos docentes, en que la implementación tecnológica va a significar una disminución del compromiso motor de los estudiantes, pues supuestamente conlleva a una reducción del volumen de actividad física y disminución en su naturaleza ludo-práctica o su tradición experiencial. Contradictoriamente con ello la situación actual ha obligado a aplicar la modalidad virtual en las clases de Educación Física, por lo que la aplicación de estrategias didácticas que respeten los fundamentos pedagógicos y al mismo tiempo promuevan una motivación en los estudiantes y que genere resultados satisfactorios en el proceso de enseñanza- aprendizaje de la disciplina está siendo en los últimos meses bien mirada por la comunidad científica.

En este ámbito autores como Cazorla, Ibánez, (2018) han señalado que se debe considerar que de una forma u otra, las TICs ya se están utilizando para realizar actividad física, y pone como ejemplo, la utilización de smartphone para obtener datos informativos sobre la práctica de esta actividades, para conocer los esfuerzos realizados en las caminatas diarias y su equivalente en gastos calóricos entre otros, aseverando sobre diferentes aspectos positivos que tienen las TICs, que permiten ampliar las posibilidades metodológicas en la enseñanza de la disciplina y de la buena acogida que se tiene su uso, por parte de los estudiantes.

No obstante, si es importante remarcar que quizás en mayor grado en comparación a otras disciplinas, el docente de Educación Física en la modalidad virtual, necesita de una adecuada preparación, dado que en ella deben confluir los aspectos relacionados propiamente con los fundamentos teóricos-prácticos de la disciplina de Educación Física, así como la utilización efectiva de las herramientas tecnológicas y los espacios virtuales asociados a esta modalidad, para lo cual requiere de un alto grado de competencias y desempeño.

Ello ha sido compartido por Becerril, et al., (2015), al señalar que el docente debe ser capaz de implementar estrategias de enseñanza y aprendizaje que posibiliten el trabajo colaborativo y la construcción en una comunidad virtual de aprendizaje.

Con respecto a este tema, Romero, Kabusch, (2017), plantearon que la inclusión de las tecnologías de la información (TICs) para abordar situaciones en el sistema educativo, no solo está referido a la incorporación como tal de las mismas al proceso educativo, sino que debe constituir un reflejo de la institucionalidad de logros en la construcción de los conocimientos.

Otros autores validan estos conceptos, tal como Pannesi, (2011), indica que la Educación Física debe aprovechar las Tecnologías de la Información y Comunicación para lograr ambientes enriquecedores de aprendizaje que haga que sea más atractiva su enseñanza. De igual manera, Barahona, (2012), explica que el uso de la modalidad virtual en Educación 
Física, o sea de las Tecnologías de la Información y la Comunicación, debe ser capaz de estimular los principios del desarrollo al máximo en los estudiantes del potencial físico, intelectual y socio-afectivo.

Considerando y reflexionado sobre los aportes precedentes resulta básico resumir algunas ideas generales que forman parte concluyente del análisis realizado:

- Se demuestra la falsedad de la incompatibilidad del uso de la modalidad virtual, o sea de las TICs, en el proceso de enseñanza- aprendizaje de la Educación Física, bajo la creencia de que la implementación tecnológica va a significar una disminución del compromiso motor de los estudiantes, pues supone una reducción del volumen de actividad física, su naturaleza ludo-práctica o su tradición experiencial. En contraposición podemos encontrar diversos estudios que validan la contribución de diferentes aspectos positivos de las TICs, que permite ampliar las posibilidades metodológicas en la enseñanza de la disciplina y la buena acogida por parte de los estudiantes.

- Es un criterio generalizado, que la modalidad virtual exige del docente un alto nivel de desempeño y competencias, unido a una adecuada preparación, dado que en ella deben confluir tanto los aspectos relacionados propiamente con los fundamentos pedagógicos y teóricos-prácticos de la disciplina de Educación Física, así como la utilización efectiva de las herramientas tecnológicas y los espacios virtuales asociados a esta modalidad.

- De igual manera se está de acuerdo con que la Educación Física debe aprovechar las Tecnologías de la Información y Comunicación para lograr ambientes enriquecidos de aprendizaje, de manera que se haga más atractiva su enseñanza y se contribuya a que se genere un cambio metodológico, que varíe las prácticas pedagógicas tradicionales, fundamentadas en la rigidez de los contenidos, el monologo docente y el exacerbado protagonismo del libro de texto.

- Se concuerda en que el uso de la modalidad virtual, o sea de las Tecnologías de la Información y la Comunicación, debe estimular los principios del desarrollo al máximo en los estudiantes del potencial físico, intelectual y socio-afectivo.

- Se coincide con los autores precedentes en que, en la modalidad virtual, lo primordial no es la tecnología como tal, sino el uso que podemos hacer de ella dentro del proceso educativo. 
Derivado del análisis realizado los autores del presente trabajo exponen los siguientes resultados:

1. Se realizó la revisión de 25 fuentes, lográndose la identificación de 15 estudios potencialmente relevantes que contribuyeron a sistematizar las estrategias seguidas sobre la importancia de la aplicabilidad de los fundamentos pedagógicos de la Educación Física para su enseñanza-aprendizaje mediante la modalidad virtual.

2. El análisis de los estudios precedentes devela el reto que ha significado la introducción en los contextos actuales de la modalidad virtual en las clases de Educación Física, precisamente por estar contemplada la Educación Física dentro del grupo de asignaturas prácticas y las limitadas competencias de los docentes para desarrollar el proceso de enseñanza -aprendizaje en este nuevo escenario, sin embargo ha quedado demostrado y se concuerda en que su desarrollo presupone en primer término la implementación y seguimiento de los fundamentos pedagógicos y el desarrollo de las competencias necesarias por parte de los docentes, pero a su vez se confirma su aplicabilidad en condiciones de modalidad virtual.

3. Dada las bondades que tienen las TICs y las oportunidades que ofrecen las herramientas y recursos tecnológicos, se ha podido dar continuidad en tiempos de Covid 19 al proceso de enseñanza - aprendizaje e incidir de manera positiva en el desarrollo de otras competencias tanto en los docentes como en los estudiantes.

\section{Conclusiones.}

- El análisis de las teorías precedentes confirma la importancia de los fundamentos pedagógicos que sustentan la Educación Física y su utilidad para el desarrollo del proceso de enseñanza-aprendizaje mediante la modalidad virtual, sin dejar de ser conscientes que lo primordial no es la tecnología como tal, sino el uso que podemos hacer de ella dentro del proceso educativo.

- Independientemente a ello migrar de la modalidad presencial a la virtual, ha constituido un gran reto, provocando la necesidad de potenciar en tiempo récord el desarrollo de competencias y habilidades didácticas -metodológicas en los docentes y estudiantes, los cuales han tenido que hacer más coherente la relación entre su praxis diaria y los fundamentos pedagógicos para el desarrollo del proceso de enseñanza aprendizaje en esta disciplina.

- El uso de las TICs, ha permitido dar continuidad al proceso de enseñanza-aprendizaje de esta disciplina, favoreciendo cumplir con los objetivos instructivos y educativos de la Educación Física, sin dejar de ser conscientes que lo primordial no es la 
tecnología como tal, sino el uso que podemos hacer de ella dentro del proceso educativo.

\section{Referencias bibliográficas.}

Alfonso A, Enríquez L, Alcívar L (2020)- Estrategias didácticas para la efectividad de la educación física: un reto en tiempos de confinamiento. Educación Física: Efectividad en tiempos de confinamiento social. Revista Electrónica Formación y Calidad Educativa (REFCalE). ISSN 1390-9010.

Barahona, J (2012)- La enseñanza de la Educación Física implementada con TIC- Revista Educación física y deporte, n. 31-2, 1047-1056, 2012, Funámbulos Editores.

Barrachina j \& Blazco J, (2012)- Análisi del desenvolupament de les competéncies básiques en el currículum d'educació física en l'ESO a la Marina Baixa. Un estudi de cas. Apunts, 110, 36-44.

Becerril C, Sosa G, Delgadillo M y Torres Sandra (2015)- Competencias Básicas de un Docente Virtual. Revista de Sistema y Gestión Educativa. Septiembre 2015 Vol 2 No. 4 882-887. ECORFAN-Bolivia- ISSN-2410-3977.

Cazorla D, Ibánez, (2018)- Rediseño del proceso formativo mediante la introducción de las TIC. Diseño, desarrollo e implementación de un Entorno Virtual de EnseñanzaAprendizaje en el área de Educación Física- Trabajo Final de Master. Máster Oficial Interuniversitario en Tecnología Educativa: e-Learning y Gestión del Conocimiento. Universitat Rovira Virgili-Universitat de les Illes Balears-Universitat de LleidaUniversidad de Murcia.

Díaz, J. (2011). La Educación Física y sus maestros en el marco de una enseñanza competencial. pp. 15-30. En Educación Física.

Fariña-Vargas, E., González-González, C., \& Área Moreira, M. (2013). ¿Qué uso hacen de las aulas virtuales los docentes universitarios? RED. Revista de Educación a Distancia. (35), pp. 1-13.

Ferreres, C. (2011). La integración de las tecnologías de la información y de la comunicación en el área de la Educación Física de secundaria: Análisis sobre el uso, nivel de conocimientos y actitudes hacia las TIC y de sus posibles aplicaciones educativas. Tesis Doctoral, Universitat Rovira i Virgili. Departamento de Pedagogía). TDX/TDR database. En: http://www.tesisenred. net/handle/10803/52837

Florez Ramos, V. (2015). 2015: año para fortalecer la formación presencial. Virtualmente. Revista de la Facultad de Estudios en Ambientes Virtuales. pp.1- 18.

García M, (2010)- Aportaciones al desarrollo de competencias básicas desde el área de Educación Física-Wanceulen-E.F.digital, 6, 52-56.

Maqueira; G (2018), Pedagogía no directiva, integración de saberes y aprendizajes significativos en Educación Física: Aproximaciones y reflexiones. Revista Olimpia. 
ISSN: 2602-8085

Vol.15. No.50, $\quad$ Pág $\quad 36-44 \quad$ olimpia@udg.co.cu https://revistas.udg.co.cu/index.php/olimpia/article/view/86

Monzonis N. (2015)- La Educación Física como elemento de mejora de la Competencia Social y Ciudadana- Investigación acción en torno a la aplicación de un programa basado en la prevención y resolución de conflictos desde el área de la Educación Física. - Tesis doctoral-Programa de Doctorado Activitat Física, Educació Física i SportUniversitat de Barcelona.

Moreira F. (2018). Uso de las aplicaciones de mensajería instantánea como potencial distractor del proceso de enseñanza/aprendizaje en programas de posgrado. Revista FADMI: Administración y Tecnología, 2(2), 22-25.

Moreno, P., \& Trigo, E. (2017). Las TIC y las TAC al servicio de la educación: Una introducción a los mapas conceptuales y la toma de apuntes. Revista de estudios socioeducativos: RESED, $\quad$ 5, 103 . https://doi.org/10.25267/Rev_estud_socioeducativos.2017.i5.09.

Pannessi, M. (2011). ¡Devolvamos el protagonismo al alumnado! En: Hernández, J; Pennessi, M; Sobrino, D; Vázquez (Coord.). Experiencias educativas en las aulas del siglo XXI. Innovación con TIC. (pp. 145-149). Madrid: Editorial Ariel.

Reyes M \& Fuentes M, (2011). Competéncia d’aprende a aprende, En A. Zabala (Ed.), Qué, quam i com enseyar competéncies básiques a primária. (pp. 77-86). Barcelona:Graó.

Rodríguez D, (2010)- El futbol como herramienta para el trabajo de los valores y las actitudes en la ESO según las Competencias Básicas. Retos. Nuevas tendencias en Educación Física, Deportes y Recreación, 17, 67-71.

Rodríguez N, Trybalski M, (2020)- Plan de continuidad pedagógica, educación física y estado de excepción. estudio sobre la educación secundaria en la provincia de buenos aires. Revista Tempos Espaços Educ.v.13, n. 32, e-14088, jan./dez.2020. 2020 -ISSN 2358-1425. Doi:http://dx.doi.org/10.20952/revtee.v13i32.14088.

Rodríguez O, Peña A, Reginfo J, Rojas N, (2015)- La lúdica y las TIC en Educación Física liceo técnico comercial. Trabajo de grado para optar al título de Especialista en Pedagogía de la LúdicaFundación Universitaria "Los Libertadores". Facultad de Ciencias de la Educación. Valle del Cauca. Colombia. URI: http://hdl.handle.net/11371/183.

Rojas M, (2018)- Aula virtual para el desarrollo de las competencias específicas para el área de Educación Física, Recreación y Deportes en el grado séptimo la IE Álvaro Gómez Hurtado jornada tarde. Trabajo de grado presentado para optar al Título de Especialista en Informática para el Aprendizaje en Red. Fundación Universitaria los Libertadores. Facultad de Ciencias de la Educación. Programa de especialización en informática para el aprendizaje red. Bogota D.C- Colombia.

Romero, C.; Kabusch, A. (2017). Implementación de entornos virtuales de aprendizaje en el desarrollo de la práctica docente en Educación Física. $12^{\circ}$ Congreso Argentino de 
Educación Física y Ciencias, 13 al 17 de noviembre 2017, Ensenada, Argentina. Educación Física: construyendo nuevos espacios. EN: Actas (2017). Ensenada: Universidad Nacional de La Plata. Facultad de Humanidades y Ciencias de la Educación. Departamento de Educación Física. En Memoria Académica. Disponible en: http://www.memoria.fahce.unlp.edu.ar/trab_eventos/ev.10293/ev.10293.pdf.

Ruiz J, (2010). Las actividades físicas cooperativas en un currículo articulado a través de competencias: implicaciones y aportaciones educativas. "La Peonza". Revista de Educación Física para la paz, 5, 3-18.

Salinas, A. (2017). Información sobre qué es, quién creo y cómo funciona la aplicación Whatsapp. Blog de Marketing Digital y Estrategias de Contenido. https://mott.marketing/informacion-sobre-que-es-quien-creo-y-como-funciona-laaplicacionwhatsapp/.

Sánchez E, Ávila C, García D y Bravo W, (2020)- El proceso de enseñanza-aprendizaje de la Educación Física en época de pandemia. Artículo de investigación -Ciencias Técnicas y Aplicadas. Polo del Conocimiento. Pol. Con. (Edición núm. 52) Vol. 5, No 11 noviembre 2020, pp. 455-467 ISSN: 2550 - 682X DOI: 10.23857/pc. v5i11.1936

Úbeda, J. y Molina, J. (2016). El blog como herramienta didáctica en educación física: la percepción del alumnado. Apunts. Educación Física y Deportes, 126, 37-45. Recuperado de http://www.raco.cat/index.php/ApuntsEFD/article/view/316519

\section{【L Ciencia}




\section{PARA CITAR EL ARTÍCULO INDEXADO.}

Carrasco Coca, O. R., Caicedo Merizalde, J. G., Savedra Valdiviezo, O. A., \& Ochoa Sangurima, V. L. (2021). Fundamentos Pedagógicos para la enseñanza-aprendizaje de la Educación Física en Modalidad Virtual: Un reto actual. Ciencia Digital, 5(1), 232-251. https://doi.org/10.33262/cienciadigital.v5i1.1542

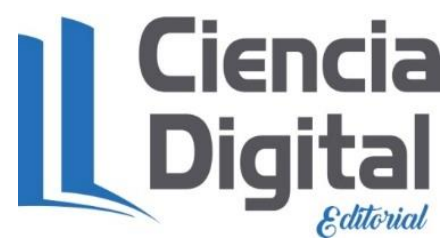

El artículo que se publica es de exclusiva responsabilidad de los autores y no necesariamente reflejan el pensamiento de la Revista Ciencia Digital.

El artículo queda en propiedad de la revista y, por tanto, su publicación parcial y/o total en otro medio tiene que ser autorizado por el director de la Revista Ciencia Digital.
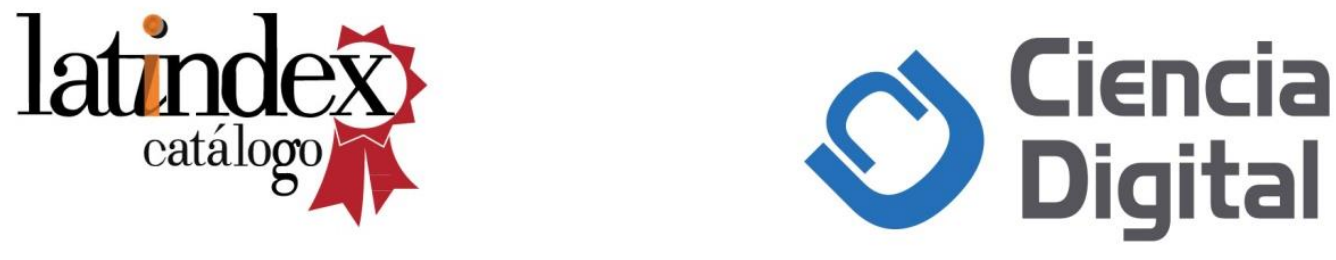